\title{
Analysis of PVA/AA based photopolymers at the zero spatial frequency limit using interferometric methods
}

\author{
Sergi Gallego, ${ }^{1, *}$ Andrés Márquez, ${ }^{1}$ David Méndez, ${ }^{1}$ Manuel Ortuño, ${ }^{1}$ Cristian Neipp, ${ }^{1}$ \\ Elena Fernández, ${ }^{2}$ Inmaculada Pascual, ${ }^{2}$ and Augusto Beléndez ${ }^{1}$ \\ ${ }^{1}$ Departamento de Física, Ingeniería de Sistemas y Teoría de la Señal, Universidad de Alicante, Ap. 99, 03080 Alicante, Spain \\ ${ }^{2}$ Departamento de Óptica, Universidad de Alicante, Ap. 99, 03080 Alicante, Spain \\ ${ }^{*}$ Corresponding author: sergi.gallego@ua.es
}

Received 6 February 2008; revised 16 April 2008; accepted 16 April 2008;

posted 16 April 2008 (Doc. ID 92536); published 2 May 2008

\begin{abstract}
One of the problems associated with photopolymers as optical recording media is the thickness variation during the recording process. Different values of shrinkages or swelling are reported in the literature for photopolymers. Furthermore, these variations depend on the spatial frequencies of the gratings stored in the materials. Thickness variations can be measured using different methods: studying the deviation from the Bragg's angle for nonslanted gratings, using MicroXAM S/N 8038 interferometer, or by the thermomechanical analysis experiments. In a previous paper, we began the characterization of the properties of a polyvinyl alcohol/acrylamide based photopolymer at the lowest end of recorded spatial frequencies. In this work, we continue analyzing the thickness variations of these materials using a reflection interferometer. With this technique we are able to obtain the variations of the layers refractive index and, therefore, a direct estimation of the polymer refractive index. (C) 2008 Optical Society of America

OCIS codes: $160.0160,160.5470,160.4670$.
\end{abstract}

\section{Introduction}

Analyzing the behavior of a photopolymer as an optic storage medium is a complicated task. Normally, these materials are used in holographic applications, where high values of spatial frequencies are recorded. In this range of frequencies many processes are involved in the hologram formation, such as species diffusion, nonlocal polymerization (due to the finite size of polymer chains), and shrinkage or swelling (volume changes) [1]-8]. In this sense, the variations in the estimation of the monomer diffusion affect significantly the values obtained for other material parameters, making it difficult to calculate them separately. In particular, it is interesting to analyze the material behavior at very low spatial frequencies, since at these frequencies monomer diffusion does not play an important role and even disappears at the zero spatial frequency limit. Moreover, there are practical applications where these materials are used

0003-6935/08/142557-07\$15.00/0

(C) 2008 Optical Society of America to store very low spatial frequencies [9-[1], as in the case of diffractive optical elements recorded onto polyvinyl alcohol/acrylamide (PVA/AA) based photopolymers. This paper is the continuation of a previous work [12], where some compositions of PVA/AA based photopolymer were studied at the zero frequency limit using a transmission interferometric setup. The phase shift between the exposed and nonexposed areas in the transmission interferometric case is due to two different effects: monomer polymerization in the exposed zones and thickness variations when polymerization occurs. Since we want to study these two effects separately, we can use a reflection interferometric setup to analyze the thickness variation during exposition at the zero spatial frequency limit. In this case, the thickness variations of the material $(\Delta d)$ can be expressed as follows:

$$
\Delta d=\frac{\Delta \Phi \lambda \cos \alpha}{4 \pi}
$$

where $\Delta \Phi$ is the phase shift (in radians) between the exposed and nonexposed zones along the whole 
round-trip forward reflected paths, and $\lambda$ and $\alpha$ are the wavelength and the incident angle of the reading beam, respectively. Usually, shrinkages around $8 \%$ are reported in the literature for different polymer formulations [13, 14]. Shrinkage of $0.5 \%$ is defined as the upper limit for commercial viability of a recording medium 15, 15, 16]. Nevertheless, it is important to note there are other cases where relief profiles (and, so, higher shrinkage or swelling) are required, i.e., liquid crystal related applications [17].

Once the thickness variation is evaluated, it is possible to calculate the refractive index difference between the exposed and nonexposed zones. If we know the volume fractions of each photopolymer component, we can estimate the polymer (polyacrylamide chains in this case) refractive index. This estimation is an important drawback to model the hologram formation in photopolymers [18-20]. In this sense, it is interesting to mention that the average polymer chain length (if the length of the chain is longer the polymer refractive index increases) depends on many factors, such as the layer humidity, the crosslinker used, and the recording intensity.

In order to analyze the refractive index variations, we have followed some previous studies [21,22] where the authors assume that there is no change in the total volume. In our case, if we want to make this assumption, we have to consider the holes inside the material (some molecules of air and vacuum are dissolved into the photopolymer solution). As monomer chains start to grow, the photopolymer layer becomes more compact and air molecules escape out of the "solid" layer and shrinkage appears. In other words, when exposure finishes, we can consider that the material is divided in two different zones: the new "solid" layer (thinner than the initial one and so with a lower hole volume fraction) and a new zone composed only of holes. Then we can consider

$$
\phi^{(m)}+\phi^{(p)}+\phi^{(h)}+\phi^{(b)}=1
$$

where $\phi^{(m)}, \phi^{(p)}, \phi^{(h)}$, and $\phi^{(b)}$ are the volume fractions of the monomer, the polymer, the holes, and the background (PVA, triethanolamine, and dye), respectively. While the collapse of holes will result in a reduction in the overall volume, the total volume fraction is, by definition, conserved. The total refractive index $(n)$ can then be expressed using the Lorentz-Lorenz relation [21,22]:

$$
\begin{aligned}
\frac{n^{2}+1}{n^{2}+2}= & \frac{n_{m}^{2}-1}{n_{m}^{2}+2} \phi^{(m)}+\frac{n_{p}^{2}-1}{n_{p}^{2}+2} \phi^{(p)}+\frac{n_{b}^{2}-1}{n_{b}^{2}+2} \phi^{(b)} \\
& +\frac{n_{h}^{2}-1}{n_{h}^{2}+2} \phi^{(h)}
\end{aligned}
$$

where $n_{m}, n_{p}, n_{b}$, and $n_{h}$ are the refractive indices of monomer, polymer, background, and holes, respectively.
Thus, if we consider Eqs. (2) and (3) together:

$$
\begin{aligned}
\frac{n^{2}+1}{n^{2}+2}= & \left(\frac{n_{m}^{2}-1}{n_{m}^{2}+2}-\frac{n_{b}^{2}-1}{n_{b}^{2}+2}\right) \phi^{(m)}+\left(\frac{n_{p}^{2}-1}{n_{p}^{2}+2}-\frac{n_{b}^{2}-1}{n_{b}^{2}+2}\right) \phi^{(p)} \\
& +\left(\frac{n_{h}^{2}-1}{n_{h}^{2}+2}-\frac{n_{b}^{2}-1}{n_{b}^{2}+2}\right) \phi^{(h)}+\frac{n_{b}^{2}-1}{n_{b}^{2}+2} .
\end{aligned}
$$

The volume fraction is given by $\phi_{i}=x_{i} \nu_{i} / \Sigma x_{i} \nu_{i}$, where $x_{i}$ is the mole fraction and $\nu_{i}$ is the molar volume of the $i$ th component. To calculate the refractive index variation $\left(n_{1}\right)$ as a function of the variations in the volume fraction of each component, we can calculate the following approximation, where we assume that $n_{h}=1$ (i.e., in vacuum),

$$
\begin{aligned}
n_{1}= & \frac{\left(n_{\mathrm{dark}}^{2}+2\right)^{2}}{6 n_{\mathrm{dark}}}\left[-\left(\frac{n_{m}^{2}-1}{n_{m}^{2}+2}-\frac{n_{b}^{2}-1}{n_{b}^{2}+2}\right) \phi_{1}{ }^{(m)}\right. \\
& \left.+\left(\frac{n_{p}^{2}-1}{n_{p}^{2}+2}-\frac{n_{b}^{2}-1}{n_{b}^{2}+2}\right) \phi_{1}{ }^{(p)}+\left(\frac{n_{b}^{2}-1}{n_{b}^{2}+2}\right) \phi_{1}{ }^{(h)}\right],
\end{aligned}
$$

where $n_{\text {dark }}$ is the average refractive index of the material before exposure, $n_{p}$ is the polymer refractive index, $n_{m}$ is the monomer refractive index, $n_{b}$ is the binder refractive index, and $\phi_{1}{ }^{(i)}$ is the volume fraction variation of the $i$ th component. The effect of hole generation and collapse described in [21] does not affect when we study the zero spatial frequency limit due to the uniformity in the exposition.

In the material used in this work, the different refractive indexes have the following values: $n_{m}=$ $1.486, n_{b}=1.474$, and $n_{\text {dark }}=1.478$. These values were obtained using the Lorentz-Lorenz equation and the method described in [21,23]. The calculations are based on refractometer measurements using water solutions. To estimate two different polymer refractive indices,primeave used two different compositions: one without BMA (N,N'-methylenebisacrylamide) and the other with BMA as crosslinker (in this case the polymer refractive index will be higher than in the previous case [24]).

\section{Experimental}

We have designed the experimental setup presented in Fig. 1 1 to measure the phase shift (due to the thickness variation in exposed zones) as a function of the exposure energy. The setup has two arms with an angular separation of $14.4^{\circ}$. One is used to expose the recording material, whereas the second arm is the interferometer used to measure, in real-time, the phase shift. The recording material is oriented with an angle of $7.6^{\circ}$ with respect to the measuring beam. In the first arm, the exposure beam, provided by a solid-state $\mathrm{Nd}-\mathrm{YVO}_{4}$ Verdi laser with a wavelength of $532 \mathrm{~nm}$ (at this wavelength the dye presents the maximum absorption), is expanded and collimated using a spatial filter and a lens obtaining a beam with a radius of $1.5 \mathrm{~cm}$. A wave plate and a 


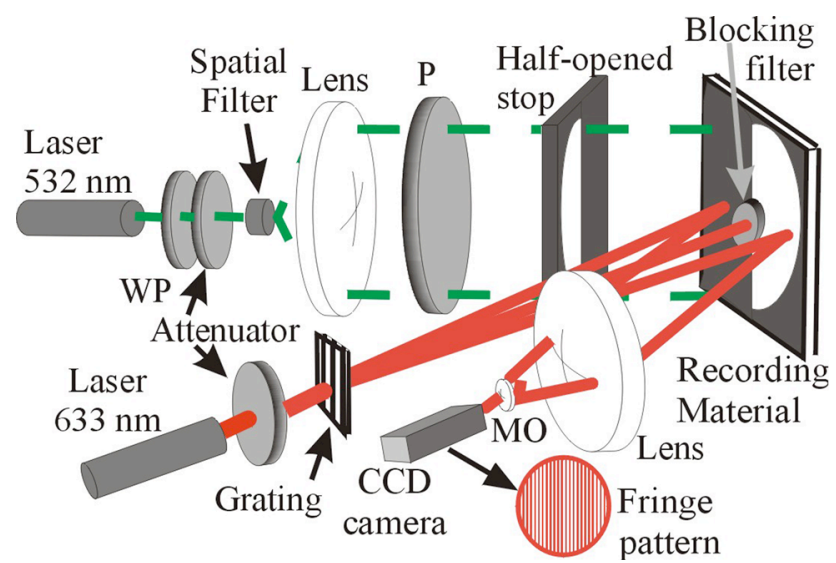

Fig. 1. (Color online) Experimental setup. The recording material is exposed with the green laser beam $(\lambda=532 \mathrm{~nm})$ and the phase shift is measured with the red beam $(\lambda=633 \mathrm{~nm})$. P is polarizer; WP is wave plate; MO is microscope objective.

neutral filter (attenuator) are added before the spatial filter to control the orientation and the intensity of the linearly polarized beam produced by the $\mathrm{Nd}-\mathrm{YVO}_{4}$ laser. A polarizer $(\mathrm{P})$, with its transmission axis oriented along the vertical of the lab, is introduced to produce a beam with TE polarization incident onto the recording material. We adjust the laser power so that the exposure intensity that impinges on the layer is $0.4 \mathrm{~mW} / \mathrm{cm}^{2}$ (this is the value corrected from the Fresnel coefficient at the airphotopolymer interface with an angle of incidence of $14.4^{\circ}$ for TE polarization). A half-opened diaphragm is used to leave an unexposed area in the photopolymer layer. Since the photopolymer does not present any absorption at $633 \mathrm{~nm}$, we use a He-Ne laser to generate an interference pattern. This technique has been successfully applied in the phase-shift characterization of liquid crystal displays (LCDs) [25]. It shows good precision, and due to its quasi-common path architecture, is a robust setup, less sensitive to changing environmental conditions, and simpler to construct than Mach-Zehnder type interferometers. We use a grating with a spatial frequency of 4 lines $/ \mathrm{mm}$ to generate a series of diffracted orders from the unexpanded $\mathrm{He}-\mathrm{Ne}$ beam; we block all the orders except -1 and +1 . One of the two orders impinges on the exposed zone (illuminated by the $\mathrm{Nd}-\mathrm{YVO}_{4}$ laser) and the other one impinges on the nonexposed zone. The distance between the two orders is about $1 \mathrm{~cm}$, enough to eliminate the influence of the monomer diffusion in the polymerization process. Once the two orders are reflected by the photopolymer, we place a lens to make them interfere. A microscope objective is used to amplify the interference pattern onto a CCD camera. This pattern is captured at specific exposure time intervals, then it is digitized and transferred to a personal computer.

Once the interference pattern has been stored, we can measure the shift with respect to the initial pattern obtained for the unexposed layer. To increase the accuracy in this calculation, we cross correlate the different interference patterns with the initial one. The cross correla- tion produces a clear peak [12]. The location of this peak for each correlated interference pattern with respect to the center of the image is equal to the shift in the fringe pattern. A full fringe shift is equal to a $2 \pi$ radians variation in the phase shift. In this experiment, as we are using a reflection interferometer, the phase shift is directly related to the thickness variation of the material. Depending on the sense of movement of the fringes (in-

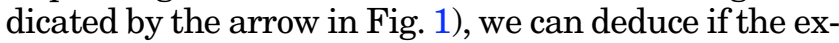
posed zone is shrinking or swelling with respect to the nonexposed one. In the case of the photopolymer studied in this paper, we obtained that the exposed zone shrinks with respect to the nonexposed area.

Material preparation is the same that was presented in the previous work [12]. The PVA/AA formulations contain a dye (yellowish eosin), a cosensitizer, which is triethanolamine (TEA), one or two monomers (AA with BMA or without it) and a polymer as a binder (PVA). In this sense, using a direct method at the zero spatial frequency limit, we have analyzed the average polymer refractive index together with the shrinkage of the layer in two different cases: with and without BMA.

\section{Results and Discussion}

In this section, we analyze the thickness variation for two different compositions, with and without crosslinker, at the zero spatial frequency limit. We study layers with a thickness of $85 \mu \mathrm{m}$.

\section{A. Layers without Crosslinker}

First, we analyze layers without crosslinker. These layers are characterized by shorter polymer chains, less energetic sensitivity, higher values of inhibition period [26-28], and lower values of diffraction efficiency when used in holography. As we have mentioned in Section 1, according to Eq. (11), from the values of phase shift between the exposed and nonexposed zones [Fig. 2(a)] calculated using a reflection interferometric setup, we can directly obtain the shrinkage of the layer during the exposition [Fig. 2(b)]. The shrinkage suffered by the sample during the first 300 seconds of exposition is around $2 \mu \mathrm{m}$ (i.e., a $2.2 \%$ change). We remark that for holographic applications, due to the shrinkage, there exists a variation of the Bragg angle (i.e., when slanted gratings are stored). It is important to note that this shrinkage value exceeds the $0.5 \%$ defined as the upper limit for commercial viablility of a holographic recording medium [15,16]. Nevertheless, these obtained photoinduced surface reliefs can be used to align liquid crystals [29,30].

Once we have calculated the shrinkage for the layers without crosslinker, we can use these data to analyze the material behavior in the transmission interferometric experiments we already discussed in [12]. In Fig. 3(a), we present the phase shift obtained between the exposed and nonexposed zones for a transmission experiment using a layer of $85 \mu \mathrm{m}$. In this case, the phase variation is due to three different effects: the shrinkage, the polymerization of acrylamide, and the decreasing of the hole volume fraction 


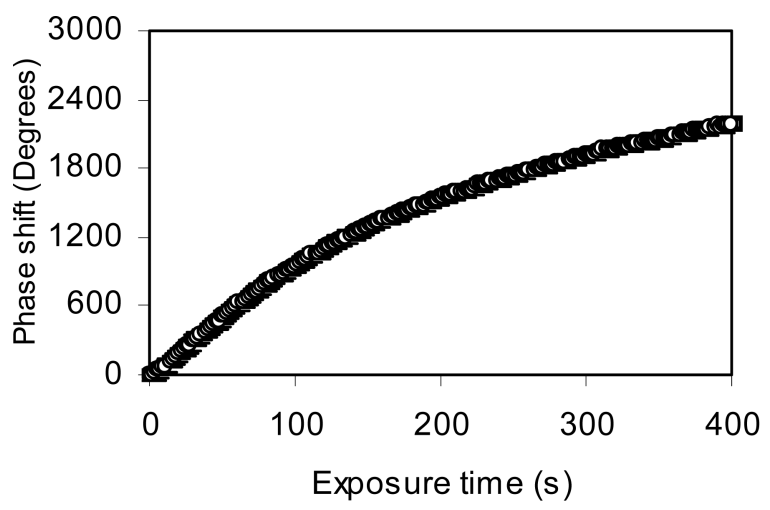

(a)

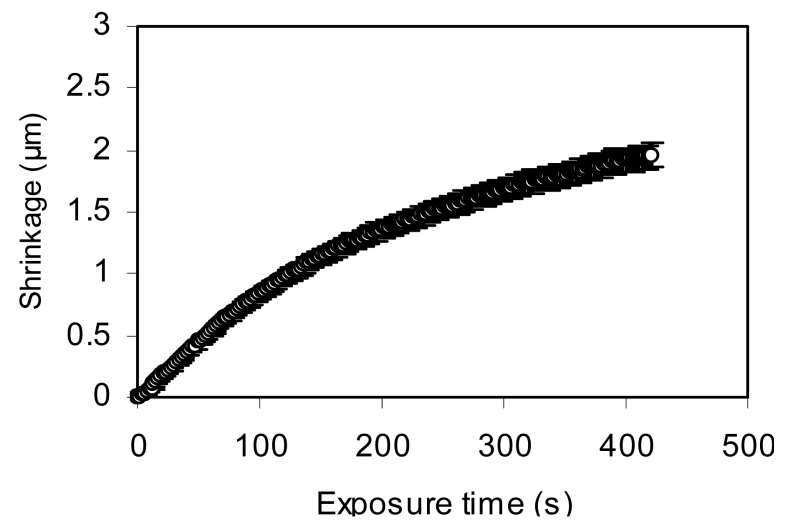

(b)

Fig. 2. (a) Phase shift as a function of the exposure time for layers without crosslinker using a reflection interferometer. (b) Layer shrinkage as a function of the exposure time for layers without crosslinker.

in the new "solid" layer, since at the zero spatial frequency limit diffusion does not take place. Therefore, if we calculate the variation due to shrinkage from the reflection data, we can obtain separately the phase shift produced by the refractive index variation. The phase shift measured in the transmission case $\left(\Delta \Phi_{t}\right)$ can be described as follows:

$$
\Delta \Phi_{t}=\frac{2 \pi}{\lambda} d(t) \cdot \Delta n \cos \alpha^{\prime}-\Delta \Phi_{\mathrm{Shr}},
$$

where $d(t)$ is the new solid layer thickness, $\alpha^{\prime}$ is the angle inside the material, and $\Delta \Phi_{\text {Shr }}$ is the phase shift due to the shrinkage and can be expresses as:

$$
\Delta \Phi_{\mathrm{Shr}}=\frac{2 \pi}{\lambda} \Delta d\left(n_{\mathrm{dark}} \cos \alpha^{\prime}-\cos \alpha\right)
$$

where $\Delta d$ is the layer shrinkage. Afterwards, it is necessary to take into account the polymerization of acrylamide together with the variation of the hole volume fraction inside the new solid layer. In this sense, it is extremely important to evaluate this last effect if we want to obtain a realistic estimation of the index variation between the exposed and nonexposed zones. Indeed, due to the reduction in the hole volume fraction, the refractive index of the new solid layer in-

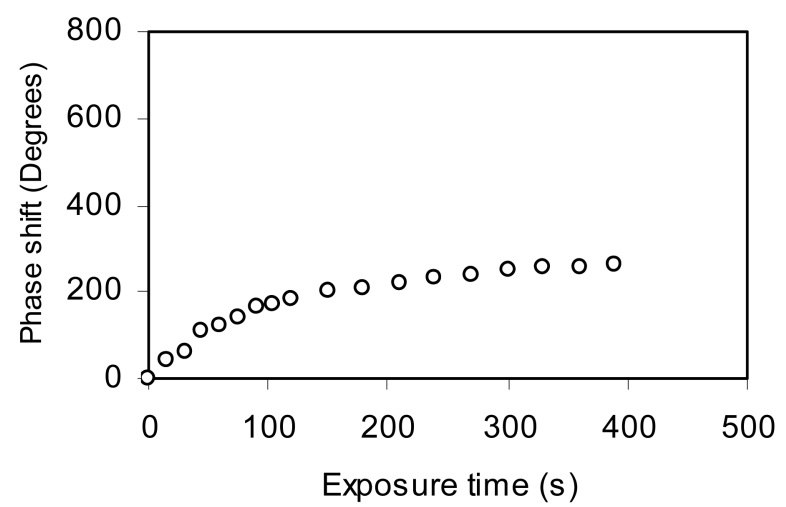

(a)

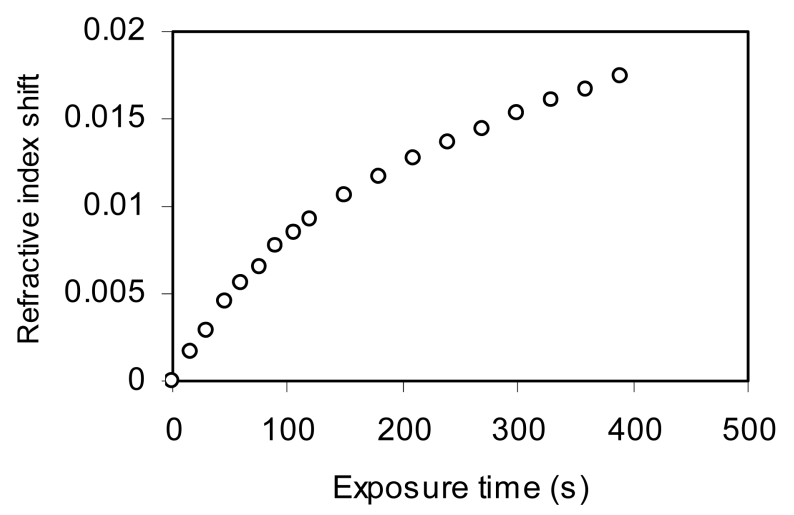

(b)

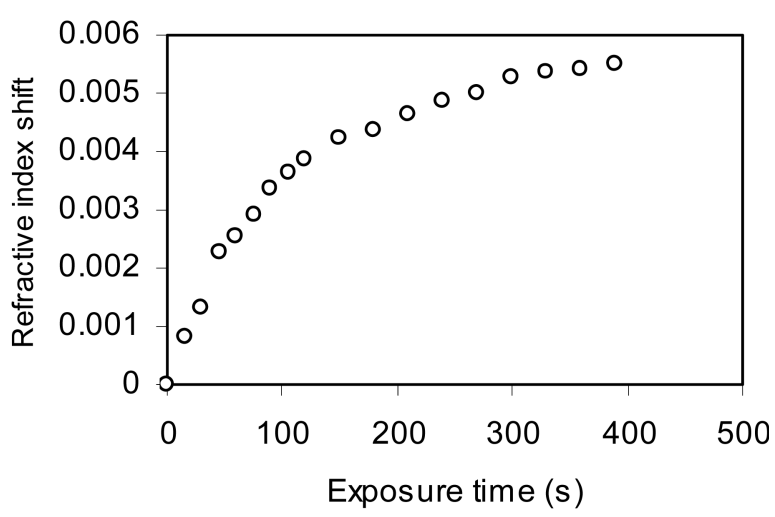

(c)

Fig. 3. (a) Phase shift as a function of the exposure time for layers without crosslinker using a transmission interferometer. (b) Refractive index shift as a function of the exposure time for the new solid layer without crosslinker. (c) Refractive index shift as a function of the exposure time for the whole layer (solid layer and holes) with crosslinker.

creases hugely [Fig. $3(\mathrm{~b})]$. The variation of the refractive index of the solid layer represented in Fig. $3(b)$ has been calculated using the following equation:

$$
\Delta n=\frac{\lambda \Delta \Phi_{t} \cos \alpha^{\prime}}{2 \pi d(t)}+\frac{\Delta d}{d(t)}\left(n_{\mathrm{dark}} \cos \alpha^{\prime}-\cos \alpha\right)
$$

This equation can be simplified for small incident angles $\left(\cos \alpha^{\prime}=\cos \alpha=1\right)$. In the case for the whole layer, 
the refractive index variation $\left(\Delta n_{w}\right)$ can be calculated as follows:

$$
\Delta n_{w}=\frac{\lambda \Delta \Phi_{t} \cos \alpha^{\prime}}{2 \pi d_{0}}
$$

Along the polymerization process, the holes are expulsed out of the solid layer and, so, its denstiy increases. Once we have characterized the different layers, it is important to remark a large phase shift between the bright and dark zones. The saturation value of the refractive index modulation (around $5 \times 10^{-3}$ [12]) is $30 \%$ higher than the typical values of refractive index modulation obtained in the holographic range (around $4 \times 10^{-3}$ at an exposure intensity of $5 \mathrm{~mW} / \mathrm{cm}^{2}$ ), where the spatial frequencies are around 1000 lines/mm. Moreover, in the case of holographic high spatial frequencies, there is also an important contribution of the free monomer coming from the nonexposed zones (monomer diffusion plays an important role for high spatial frequencies), which contributes to increase the refractive index modulation.

In order to explain these results we can consider two different (and complementary) hypotheses:

a. At low intensities $\left(0.4 \mathrm{~mW} / \mathrm{cm}^{2}\right)$ polymer chains are longer than at high intensities $\left(5 \mathrm{~mW} / \mathrm{cm}^{2}\right)$; therefore, the polymer refractive index increases [31].

b. At very low spatial frequencies, in particular in the zero spatial frequency limit, nonlocal polymerization does not take place [20]; therefore, polymer chains can become longer without invading the nonexposed zones and, so, the refractive index also increases.

\section{B. Layers with Crosslinker}

In this section we analyze the influence of BMA, acting as a crosslinker, on the material behavior. It is well-known that the crosslinkers are capable of increasing the refractive index modulation of polymers, their energetic sensitivity, and also the length of the polymer chains. Furthermore, it is interesting to study the influence of the crosslinker on the shrinkage of the layers. In Fig. 4(a), we present results of the phase shift as a function of exposure time for the reflection experiment. It is interesting to note the high values obtained in this case. These values can be explained in terms of a higher compactness produced by the BMA. As we can see in Fig. 4(b), now the shrinkage is $33 \%$ higher than in the case without crosslinker $(3 \mu \mathrm{m})$.

In our previous work [12], we have shown how the phase shift increases between the exposed and nonexposed zones when BMA is included in the material chemical composition. As we can see in Fig. 5(a), in the case of a transmission interferometer, the phase shift can achieve values around $600^{\circ}$ after 200 seconds of exposure time. Furthermore, if we study the phase shift of the new solid layer with the holes, we also obtain higher values for the refractive index [Figs. 5(b) and 5(c)].

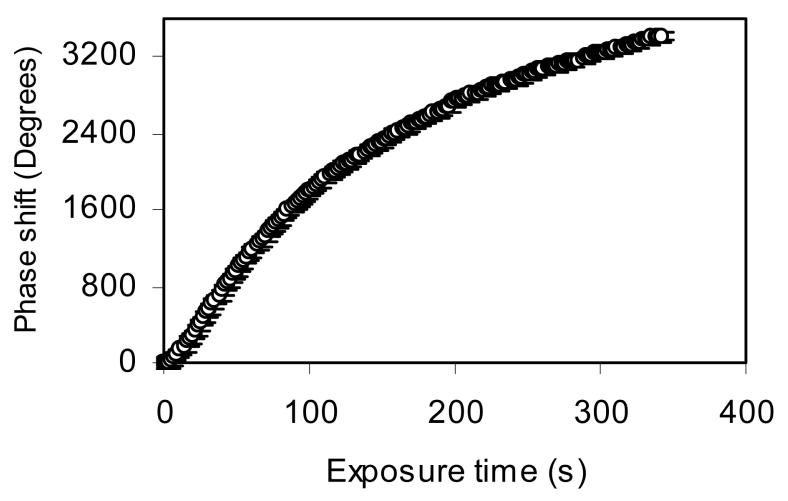

(a)

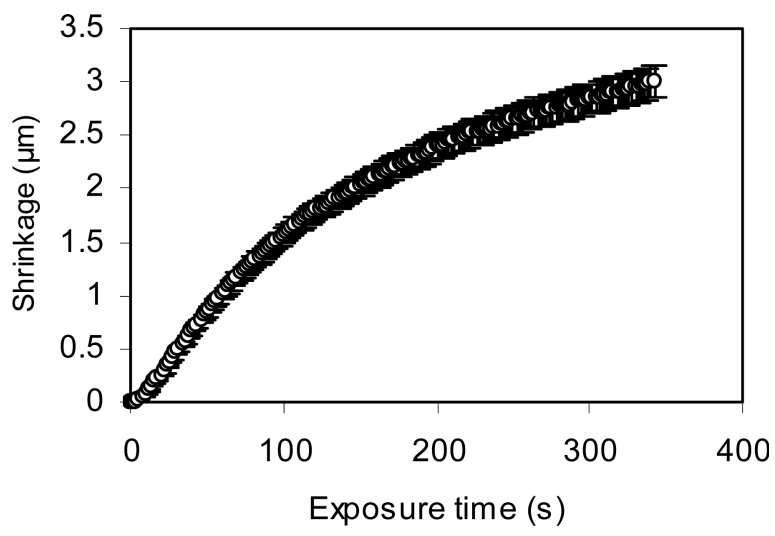

(b)

Fig. 4. (a) Phase shift as a function of the exposure time for layers with crosslinker using a reflection interferometer. (b) Shrinkage as a function of the exposure time for layers with crosslinker.

\section{Polymer Average Refractive Index Estimation}

Since diffusion does not exist at the zero spatial frequency limit, and according to Eqs. (2)-(田), we can estimate the average polymer refractive index in the material. In order to calculate an approximation of this refractive index, we will assume that when exposition finishes, all the monomers have polymerized. In this sense, it is important to obtain a precise estimation of the initial volume fraction of the acrylamide present in the "dry" layer [32]. This volume fraction can be estimated if we measure the quantity of water evaporated during the drying process. In our case, 48 hours after the layer deposition, the initial monomer volume fraction reaches a value around 0.22 . After 300 seconds of exposition, we can assume the final polymer volume fraction is similar to the initial monomer volume fraction $\left(\phi_{f}{ }^{(p)} \approx \phi_{i}{ }^{(m)}\right)$. That is, we can obtain the polymer average refractive index using Eq. (四) as follows:

$$
\begin{aligned}
\frac{n_{p}^{2}-1}{n_{p}^{2}+2}= & \frac{n_{b}^{2}-1}{n_{b}^{2}+2}+\frac{1}{\phi_{f}{ }^{(p)}}\left[\left(\frac{n_{m}^{2}-1}{n_{m}^{2}+2}-\frac{n_{b}^{2}-1}{n_{b}^{2}+2}\right) \phi_{i}{ }^{(m)}\right. \\
& \left.+\frac{n_{s}{ }^{2}+1}{n_{s}{ }^{2}+2}-\frac{n_{\text {dark }}^{2}-1}{n_{\text {dark }}^{2}+2}\right] .
\end{aligned}
$$




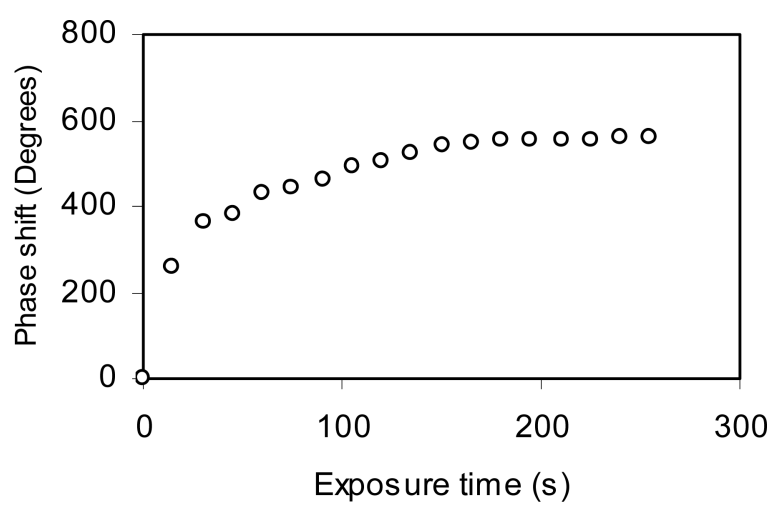

(a)

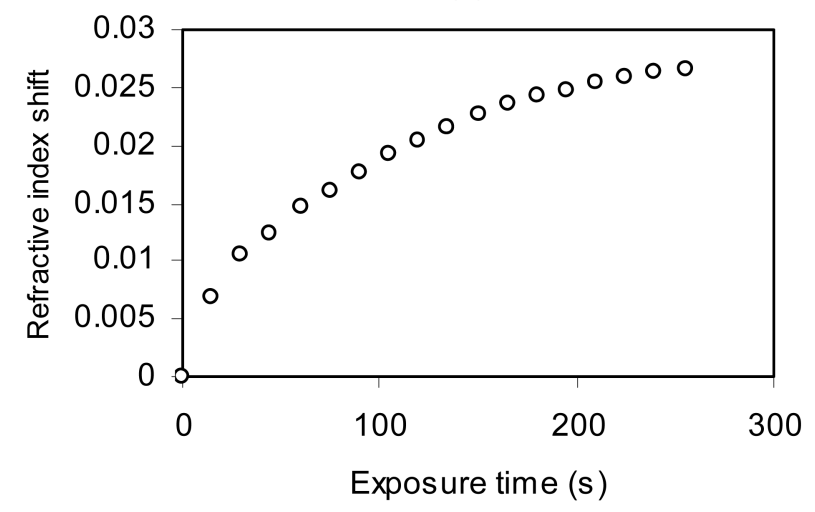

(b)

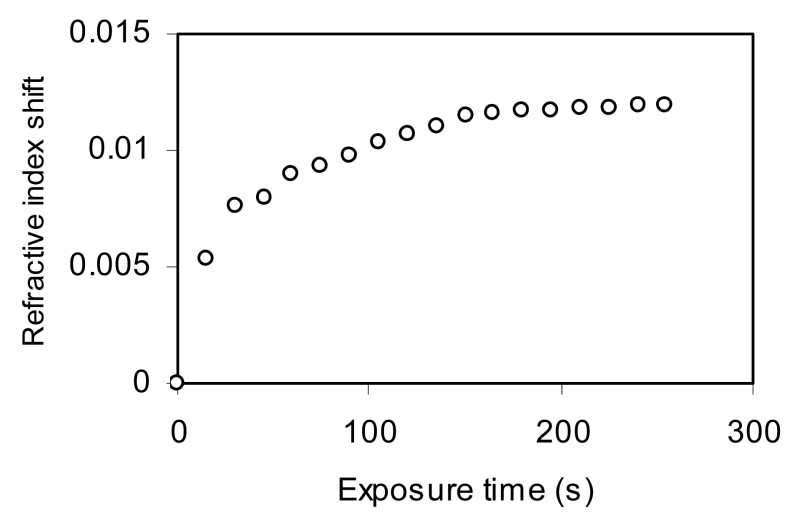

(c)

Fig. 5. (a) Phase shift as a function of the exposure time for layers with crosslinker using a transmission interferometer. (b) Refractive index shift as a function of the exposure time for layers with crosslinker when only the new solid layer is considered. (c) Refractive index shift as a function of the exposure time for layers with crosslinker when we consider the whole layer (solid layers and holes).

According to Eq. (5), we can obtain the following approximate equation:

$$
\begin{aligned}
\frac{n_{p}^{2}-1}{n_{p}^{2}+2}= & \frac{1}{\phi_{f}^{(p)}}\left[\frac{6 n_{\mathrm{dark}}}{\left(n_{\mathrm{dark}}^{2}+2\right)^{2}} n_{1}\right. \\
& \left.+\left(\frac{n_{m}^{2}-1}{n_{m}^{2}+2}-\frac{n_{b}^{2}-1}{n_{b}^{2}+2}\right) \phi_{i}{ }^{(m)}\right]+\frac{n_{b}^{2}-1}{n_{b}^{2}+2},
\end{aligned}
$$

where $n_{s}$ is the layer refractive index (taking into account the presence of holes) after exposition. Satura- tion values for the layer refractive index are $n_{s} \approx 1.489$ and $n_{s} \approx 1.484$ for layers with and without crosslinker, respectively. Using these data we can obtain values for the polymer average refractive index of $n_{p} \approx 1.537$ and $n_{p} \approx 1.514$ for layers with and without crosslinker, respectively. It is important to mention that our estimations of the polymer average refractive index are based on experimental direct measurements, whereas in holographic applications previous determinations of many parameters are needed, that is, estimations of the polymer average refractive indexes in holography are always indirect. In this sense, we must not forget that according to our hypothesis (see Section 3.A), the values obtained for the zero spatial frequency limit are always upper limits to the values for other spatial frequencies.

\section{Conclusions}

In this work, using direct measurements of the phase shift and the shrinkage of photopolymeric layers based on PVA/AA for two different compositions (with and without BMA as a crosslinker, respectively), we have estimated the polymer average refractive index at the zero spatial frequency limit. The main advantage of this method is the absence of monomer diffusion effects in the experiments carried out. We have obtained values of the shrinkage around $2 \%$ (without crosslinker) and 3\% (with crosslinker) in the layers analyzed. These values are promising so as to obtain relief structures for liquid crystal applications, but, on the other hand, are considerably high for holographic memories applications. Finally, it is important to note that at higher spatial frequencies, monomer diffusion from the dark zones to the bright ones, together with surface tension effects, can considerably affect the material volume changes. In particular, in the case of grating recorded, molecular migration reduces the shrinkage. This reduction increases with the spatial frequency and with the diffusion velocity.

This work was supported by the "Ministerio de Edicación y Ciencia" (Spain) under projects FIS200505881-C02-01 and FIS2005-05881-C02-02, and by Generalitat Valenciana under project GV06-007.

\section{References}

1. A. Márquez, C. Neipp, S. Gallego, M. Ortuño, A. Beléndez, and I. Pascual, "Edge enhanced imaging using PVA/acrylamide photopolymer gratings," Opt. Lett. 28, 1510-1512 (2003).

2. S. M. Schultz, E. N. Glytsis, and T. K. Gaylord, "Design of highefficiency volume gratings couplers for line focusing," Appl. Opt. 37, 2278-2287 (1998).

3. D. A. Waldman, C. J. Butler, and D. H. Raguin, "CROP holographic storage media for optical data storage at greater than $100 \mathrm{bits} / \mu \mathrm{m}^{2}$," Proc. SPIE 5216, 10-25 (2003).

4. W. L. Wilson, K. R. Curtis, K. Anderson, M. C. Tackitt, A. J. Hill, M. Pane, C. Stanhope, T. Earhart, W. Loechel, C. Bergman, K. Wolfgang, C. Shuman, G. Hertrich, K. Parris, K. Malang, B. Riley, and M. Ayer, "Realization of high performance holographic data storage: the in-phase technologies demonstration platform," Proc. SPIE 5216, 178-191 (2003). 
5. Holographic Data Storage, Springer Series in Optical Sciences, H. J. Coufal, D. Psaltis, and G. T. Sincerbox, eds. (Springer-Verlag, 2000).

6. C. Neipp, A. Beléndez, S. Gallego, M. Ortuño, I. Pascual, and J. T. Sheridan, "Angular responses of the first and second diffracted orders in transmission diffraction grating recorded on photopolymer material," Opt. Express 11, 1835-1843 (2003).

7. J. V. Kelly, F. T. O’ Neill, C. Neipp, S. Gallego, M. Ortuño, and J. T. Sheridan, "Holographic photopolymer materials: nonlocal polymerisation driven diffusion under non-ideal kinetic conditions," J. Opt. Soc. Am. B 22, 407-406 (2005).

8. I. Naydenova, R. Jallapuram, R. Howard, S. Martin, and V. Toal, "Investigation of the diffusion processing in selfprocessing acrylamide-based photopolymer system," Appl. Opt. 43, 2900-2905 (2004).

9. I. Pascual, A. Márquez, A. Beléndez, A. Fimia, J. Campos, and M. J. Yzuel, "Copying low spatial frequency diffraction gratings in photopolymer as phase holograms," J. Mod. Opt. 47, 1089-1097 (2000).

10. A. Márquez, J. Campos, M. J. Yzuel, I. Pascual, A. Fimia, and A. Beléndez, "Production of computer-generated phase holograms using graphic devices: application to correlation filters," Opt. Eng. 39, 1612-1619 (2000).

11. A. Márquez, C. Neipp, A. Beléndez, J. Campos, I. Pascual, M. J. Yzuel, and A. Fimia, "Low spatial frequency characterization of holographic recording materials applied to correlation,” J. Opt. A Pure Appl. Opt. 5, S175-S182 (2003).

12. S. Gallego, A. Márquez, D. Méndez, M. Ortuño, C. Neipp, M. L. Alvarez, A. Beléndez, E. Fernández, and I. Pascual, "Real-time interferometric characterization of a PVA based photopolymer at the zero spatial frequency limit," Appl. Opt. 46, 7506-7512 (2007).

13. J. E. Dietz and N. A. Peppas, "Reaction kinetics and chemical changes during polymerization of multifunctional (meth) acrylates for the production of highly crosslinked polymers used in information storage systems," Polymer 38, 3767-3781 (1997).

14. T. Endo and F. Sanda, "Ring-opening polymerization, anionic (with expansion in volume)," in Polymeric Materials Encyclopedia (CRC Press, 1996), Vol. 10, pp. 7550-7554.

15. G. Ramos, A. Álvarez-Herrero, T. Belenguer, F. del Monte, and D. Levy, "Shrinkage control in a photopolymerizable hybrid solgel material for holographic recording," Appl. Opt. 43, 4018-4024 (2004).

16. L. Dhar, M. G. Schones, T. L. Wysocki, H. Bair, M. Schilling, and C. Boyd, "Temperature-induced changes in photopolymer volume holograms," Appl. Phys. Lett. 73, 1337-1339 (1998).

17. I. Naydenova, E. Mihaylova, S. Martin, and V. Toal, "Holographic patterning of acrylamide-based photopolymer surface," Opt. Express 13, 4878-4889 (2005).
18. G. Zhao and P. Mouroulis, "Diffusion model of hologram formation in dry photopolymers materials," J. Mod. Opt. 41, 19291939 (1994).

19. S. Piazzolla and B. K. Jenkins, "First-harmonic diffusion model for holographic grating formation in photopolymers," J. Opt. Soc. Am. B 17, 1147-1157 (2000).

20. J. T. Sheridan and J. R. Lawrence, "Nonlocal-response diffusion model of holographic recording in photopolymer," J. Opt. Soc. Am. A 17, 1108-1114 (2000).

21. I. Aubrecht, M. Miller, and I. Koudela, "Recording of holographic gratings in photopolymers: theoretical modelling and real-time monitoring of grating growth," J. Mod. Opt. 45, 1465-1477 (1998).

22. J. V. Kelly, M. R. Gleeson, C. E. Close, F. T. O' Neill, J. T. Sheridan, S. Gallego, and C. Neipp, "Temporal analysis of grating formation in photopolymer using the nonlocal polymerization-driven diffusion model," Opt. Express 13, 6990-7004 (2005).

23. S. Gallego, C. Neipp, M. Otuño, A. Beléndez, E. Fernández, and I. Pascual, "Analysis of diffusion in depth in photopolymer materials," Opt. Commun. 281, 1480-1485 (2008).

24. C. Neipp, S. Gallego, M. Ortuño, A. Márquez, A. Beléndez, and I. Pascual, "Characterization of a PVA/acrylamide photopolymer. Influence of a cross-linking monomer in the final characteristics of the hologram," Opt. Commun. 224, 27-34 (2003).

25. A. Bergeron, J. Gauvin, F. Gagnon, D. Gingras, H. H. Arsenault, and M. Doucet, "Phase calibration and applications of a liquidcrystal spatial light modulator," Appl. Opt. 34,5133-5139(1995).

26. P. J. Flory, Principles of Polymer Chemistry (Cornell U. Press, 1953), pp. 161-177.

27. M. Ortuño, S. Gallego, C. García, C. Neipp, and I. Pascual, "Holographic characteristics of a $1 \mathrm{~mm}$ thick photopolymer to be used in holographic memories," Appl. Opt. 42, 7008-7012 (2003).

28. M. R. Gleeson, J. V. Kelly, C. E. Close, F. T. O' Neill, and J. T. Sheridan, "The impact of inhibition processes during grating formation in photopolymer materials," Proc. SPIE 5827, 232-243 (2005).

29. K. Pavani, I. Naydenova, S. Martin, and V. Toal, "Photoinduced surface relief studies in an acrylamide-based photopolymer," J. Opt. A Pure Appl. Opt. 9, 43-48 (2007).

30. K. Pavani, I. Naydenova, S. Martin, R. Jallapuram, R. G. Howard, and V. Toal, "Electro-optical switching of liquid crystal diffraction gratings by using surface relief effect in the photopolymer," Opt. Commun. 273, 367-369 (2007).

31. S. Gallego, M. Ortuño, C. Neipp, A. Beléndez, and I. Pascual, "Influence of the fringe visibility on the characteristics of holograms recorded in photopolymer material," Optik (Jena) 114, 401-406 (2003).

32. S. Gallego, M. Ortuño, C. Neipp, A. Márquez, A. Beléndez, and I. Pascual, "Characterization of polyvinyl alcohol-acrylamide holographic memories with a first-harmonic diffusion model," Appl. Opt. 44, 6205-6210 (2005). 